\title{
Excitons on a microscopic level: The mixed dynamic structure factor
}

\author{
Igor Reshetnyak $\odot,{ }^{1,2, *}$ Matteo Gatti, ${ }^{1,2,3}$ Francesco Sottile $\odot,{ }^{1,2}$ and Lucia Reining ${ }^{1,2}$ \\ ${ }^{1}$ Laboratoire des Solides Irradiés, École Polytechnique, CNRS, CEA/DRF/IRAMIS, \\ Institut Polytechnique de Paris, F-91128 Palaiseau, France \\ ${ }^{2}$ European Theoretical Spectroscopy Facility (ETSF) \\ ${ }^{3}$ Synchrotron SOLEIL, L'Orme des Merisiers, Saint-Aubin, BP 48, F-91192 Gif-sur-Yvette, France
}

(Received 12 July 2019; revised manuscript received 25 September 2019; published 30 October 2019)

\begin{abstract}
The dynamic structure factor of materials is proportional to their linear electronic response and it displays their excitation spectra. Usually the response is measured on the same length scale as the perturbation. Here, we illustrate that much can be gained by studying also the mixed dynamic structure factor, which connects different spatial components of perturbation and response. We extend state-of-the-art $a b$ initio calculations to access the mixed dynamic structure factor, including excitonic effects. Using bulk silicon and lithium fluoride as prototype examples, we show that these effects play a crucial role above and below the quasiparticle gap, and are needed in order to explain coherent inelastic x-ray scattering experiments. Our approach also yields important information concerning the microscopic structure of time-dependent density functional theory.
\end{abstract}

DOI: 10.1103/PhysRevResearch.1.032010

One of the key concepts in condensed matter theory is screening, the modification of a potential felt by a charge due to the rearrangement of other charges [1]. In many cases the dominant contribution to this phenomenon can be described in linear response [2]. This means that the knowledge of the response of a system to an external perturbation is entirely contained in the density-density response function $\chi$, which is determined only by the material. The poles of $\chi$ in frequency space are the excitation energies of the system. The densitydensity response function is measured directly or indirectly in many spectroscopy experiments [3], such as electron energy loss spectroscopy (EELS) [4], optical absorption [5,6], or inelastic x-ray scattering (IXS) [7], which yields the dynamic structure factor (DSF) that is proportional to the imaginary part of $\chi$. Knowing $\chi$ can also help to understand or predict effects such as a significant local rearrangement of charges as a response of the system to a perturbation, which can have dramatic effects on the structure, for example, self-trapped excitons [8]. Finally, screening is also one of the fundamental processes that govern the behavior of all many-body systems. Therefore it appears naturally as a building block in the formulation of many-body perturbation theory [9], via the screened Coulomb interaction $W=v_{c}+v_{c} \chi v_{c}$, where $v_{c}$ is the bare Coulomb interaction. The widely used $G W$ approximation (GWA) [10], for example, uses $W$ as an effective interaction.

\footnotetext{
*Present address: Chaire de Simulation à l'Echelle Atomique (CSEA), Ecole Polytechnique Fédérale de Lausanne (EPFL), CH1015 Lausanne, Switzerland.

Published by the American Physical Society under the terms of the Creative Commons Attribution 4.0 International license. Further distribution of this work must maintain attribution to the author(s) and the published article's title, journal citation, and DOI.
}

Some important features of the frequency-dependent screening are captured for the homogeneous electron gas by the Lindhard dielectric function [11] from the random phase approximation (RPA) [12], where only the classical electrostatic potential between charges is taken into account. For small momentum transfer this is sufficient to describe the long-range collective oscillations of the electron gas, called plasmons, even for simple metals and semiconductors (see, e.g., Refs. [13-17]). However, in many other cases this approximation yields unsatisfactory results. Prominent examples are materials with localized $d$ or $f$ states [18-21], or loss spectra for larger momentum transfer [13,22-24], where a shorter length scale is probed. Moreover, the RPA cannot yield bound excitons [25], which are most clearly seen in optical spectra $[26,27]$. Such many-body effects are instead captured by the Bethe-Salpeter equation (BSE) [28], a twobody Dyson equation that correlates the excited electrons and holes $[25,29]$. This equation has been successfully used to calculate optical spectra in the framework of semiempirical calculations since the 1980's [30-33], and in first-principles calculations since the 1990's [34-37]. Loss spectra for vanishing momentum transfer have been looked at more recently [38-40], and a few calculations exist for loss spectra at nonvanishing momentum transfer [41-50].

Optics, EELS and IXS probe the response of the system on the same length scale as the perturbation. Besides the spectroscopic information, this allows one also to reconstruct charge excitations as an averaged function of space and time from experimental IXS spectra [51-55]. However, in an inhomogeneous material even a spatially monochromatic perturbation creates a response on different length scales $[56,57]$, depending on the local structure of the material. Only when all these components of the response are known can one describe induced charges with spatial resolution, and can one determine important many-body effects that depend on all the components of $W$. For these reasons, it is highly 
desirable to extend state-of-the-art advanced theoretical and numerical approaches to the description of the full inhomogeneous response of materials including excitonic effects. This gives access to the mixed DSF (MDSF), which is a matrix in reciprocal space whose diagonal is the ordinary DSF [7]. The MDSF can be measured by coherent IXS (CIXS) [7]. On the theory side, some off-diagonal elements of the MDSF in silicon were calculated in the adiabatic local density approximation (ALDA) of time-dependent density functional theory (TDDFT) [58,59], which is similar to the RPA, whereas to the best of our knowledge excitonic effects have yet to be accessed.

The aim of the present Rapid Communication is to extend $a b$ initio BSE calculations to access the MDSF including excitonic effects, to benchmark against experiment, analyze and make predictions. This also leads to new guidelines for modeling approximations to TDDFT.

In periodic crystals the density-density response function can be written in reciprocal space as a function and matrix $\chi_{\mathbf{G G}^{\prime}}(\mathbf{q}, \omega)$, where $\mathbf{q}$ belongs to the first Brillouin zone and $\mathbf{G}, \mathbf{G}^{\prime}$ are reciprocal-lattice vectors. It yields the induced charge as a response to an external potential from $n_{\text {ind }}(\mathbf{q}+$ $\mathbf{G}, \omega)=\sum_{\mathbf{G}^{\prime}} \chi_{\mathbf{G G}^{\prime}}(\mathbf{q}, \omega) v_{\mathrm{ext}}\left(\mathbf{q}+\mathbf{G}^{\prime}, \omega\right)$. In a homogeneous material $\chi$ is diagonal. In an inhomogeneous material the diagonal of $\chi$ yields only a spatially averaged response, and not its local values.

To access excitonic effects, we have to extend the BSE approach to the calculation of all elements $\chi_{\mathbf{G G}^{\prime}}(\mathbf{q}, \omega)$ of the response matrix. In its state-of-the-art ab initio version this approach uses a quasiparticle (QP) approximation, and replaces the electron-hole interaction by a statically screened Coulomb interaction $W(\omega=0)$. This allows one to express $\chi$ in terms of the eigenvectors $A_{\lambda}$ and eigenvalues $E_{\lambda}$ of the two-particle Hamiltonian $\hat{H}_{\mathrm{exc}}=\hat{H}_{\mathrm{el}}+\hat{H}_{\mathrm{h}}+\hat{v}-\hat{W}$, where $\hat{H}_{\mathrm{el}}$ and $\hat{H}_{\mathrm{h}}$ contain the occupied and empty QP bands calculated in the GWA, $\hat{v}$ is the electron-hole exchange interaction due to the bare $v_{c}$, and $-\hat{W}$ is the screened direct electron-hole attraction that is responsible for excitonic effects [25]. Setting $\hat{W}=0$ corresponds to performing an RPA calculation that uses as input the $G W$ band structure: We will refer to this as RPA + $G W$. Using instead the LDA band structure as input leads to the RPA + LDA approximation, which is often simply called RPA. For a system with a gap, one can express the full matrix $\chi$ in terms of the eigenvalues $E_{\lambda}$ and eigenvectors $A_{\lambda}$ of $\hat{H}_{\mathrm{exc}}$ as

$$
\begin{aligned}
\chi_{\mathbf{G} \mathbf{G}^{\prime}}(\mathbf{q}, \omega)= & \sum_{\lambda, \lambda^{\prime}}\left[\sum_{t} A_{\lambda}^{* t}(\mathbf{q}) \tilde{\rho}_{t}^{*}(\mathbf{q}+\mathbf{G})\right. \\
& \left.\times \frac{O_{\lambda, \lambda^{\prime}}^{-1}}{\omega-E_{\lambda}(\mathbf{q})+i \eta} \sum_{t^{\prime}} A_{\lambda^{\prime}}^{t^{\prime}}(\mathbf{q}) \tilde{\rho}_{t^{\prime}}\left(\mathbf{q}+\mathbf{G}^{\prime}\right)\right],
\end{aligned}
$$

where $O_{\lambda \lambda^{\prime}}$ is the overlap matrix of the coefficients $A_{\lambda}$ that mix the transitions $t$ among QP states $\left(n \rightarrow n^{\prime}\right.$ bands and $\mathbf{k}-$ $\mathbf{q} \rightarrow \mathbf{k}$ wave vectors), with oscillator strength $\tilde{\rho}_{t}(\mathbf{q}+\mathbf{G})=$ $\left\langle\phi_{n \mathbf{k}-\mathbf{q}}\left|e^{-i(\mathbf{q}+\mathbf{G}) \cdot \mathbf{r}}\right| \phi_{n^{\prime} \mathbf{k}}\right\rangle$ [for more details, see Ref. [44], where the diagonal $\mathbf{G}=\mathbf{G}^{\prime}$ of Eq. (1) was calculated].

Off-diagonal elements of the MDSF are experimentally accessible using the transmission electron microscope [63-65] or from CIXS experiments, where the initial photon state is

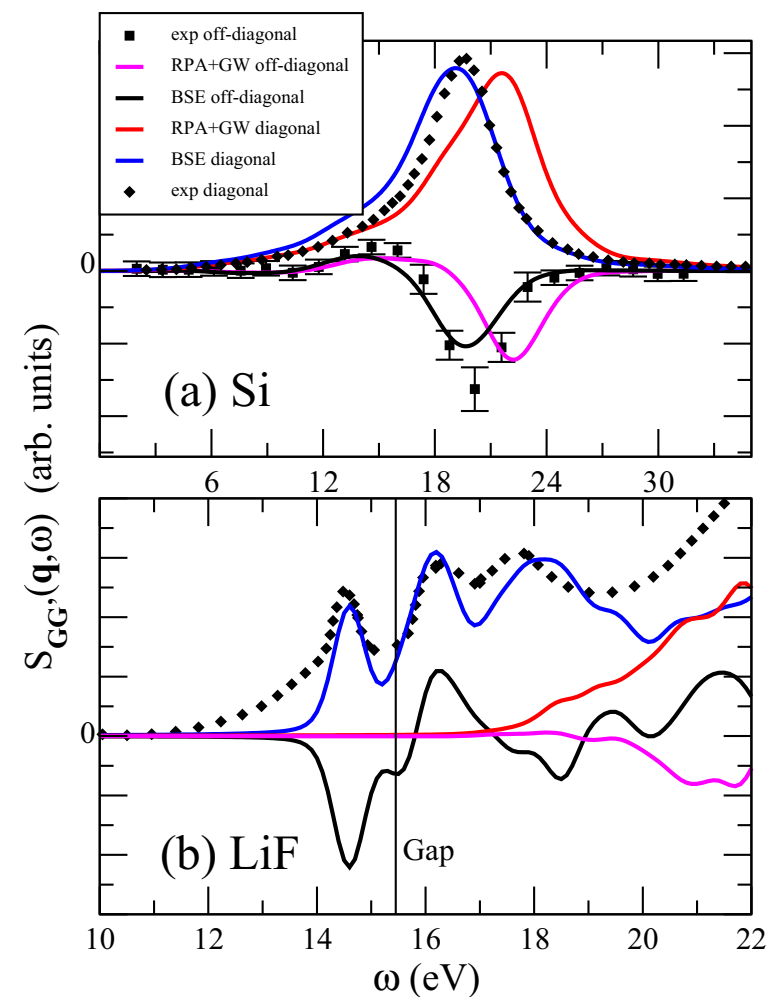

FIG. 1. Dynamic structure factor for $\mathbf{q}=(-1 / 2,-1 / 2,-1 / 2)$, $\mathbf{G}=(0,0,0), \mathbf{G}^{\prime}=(1,1,1)$ in reciprocal-lattice units for (a) $\mathrm{Si}$ and (b) LiF, obtained from the BSE (blue lines for diagonal and black lines for off-diagonal elements) and within RPA $+G W$ (red lines for diagonal and magenta lines for off-diagonal) compared to the experiments (diamonds for diagonal and squares for off-diagonal): Si CIXS [60] and IXS [22,61]; LiF EELS [62].

spatially modulated in a way that is commensurable with the intrinsic spatial modulation of the crystal [7,66-68]. In silicon, CIXS spectra were used to determine the plasmon bandgap opening at the Brillouin zone boundary due to the periodic lattice potential $[60,69-72]$. This allows us to benchmark our theoretical development against experiment for silicon, before making a prediction for the case of a strongly bound exciton in LiF.

In both these centrosymmetric crystals (see Ref. [73] for the general formulation) the MDSF $S_{\mathbf{G G}^{\prime}}(\mathbf{q}, \omega)$ is real valued and related to $\chi$ via $S_{\mathbf{G G}^{\prime}}(\mathbf{q}, \omega)=-\left[\operatorname{Im} \chi_{\mathbf{G G}^{\prime}}(\mathbf{q}, \omega)+\right.$ $\left.\operatorname{Im} \chi_{\mathbf{G}^{\prime} \mathbf{G}}(\mathbf{q}, \omega)\right] /(2 \pi)$. Figure 1(a) shows the calculated $S_{\mathbf{G G}^{\prime}}(\mathbf{q}, \omega)$ of $\mathrm{Si}$ at $\mathbf{q}=(-1 / 2,-1 / 2,-1 / 2), \mathbf{G}=(0,0,0)$ with either the diagonal element $\mathbf{G}^{\prime}=\mathbf{G}$ or the off-diagonal one with $\mathbf{G}^{\prime}=(1,1,1)$, compared to the experimental results from Refs. $[22,60,61,74]$. The diagonal element is characterized by a broad plasmon peak. As it is found in general [38], the RPA $+G W$ calculation overestimates the plasmon energy, and the electron-hole attraction yields a shift to lower energies, close to $20 \mathrm{eV}$, in good agreement with experiments [22,61]. The off-diagonal elements of $S_{\mathbf{G G}^{\prime}}(\mathbf{q}, \omega)$ look qualitatively different from the diagonal ones. In particular, their spectrum can even be negative $[58,59]$. Our results show that many-body effects act in a similar way as in the diagonal elements: Compared to experiment, the RPA $+G W$ gives a qualitatively correct description, but it overestimates the 
energy of all structures seen in experiment. The electron-hole attraction in the BSE shifts spectral weight to lower energies, so that there is a first peak at $13.8 \mathrm{eV}$ and a dip at $19.6 \mathrm{eV}$. Moreover, the first peak is sharper in BSE than in RPA $+G W$. This brings both the spectral shape and the position of the peaks much closer to experiment [60] than the RPA $+G W$ results. One may find it intuitive that the BSE acts in a similar way on diagonal and off-diagonal elements. However, this is far from trivial: Excitonic effects in silicon are interference effects, based on a subtle interplay of intensities and phases, not simply a shift of energies [35,75]. Without knowing the result, one could not have predicted the picture of Fig. 1(a).

The very good agreement between theory and experiment for silicon, and the significant excitonic effects on offdiagonal elements that emerge from the results, give strong motivation to explore and predict spectra for a material with much stronger excitonic effects. Figure 1(b) shows the calculated MDSF of LiF for the same choice of $\mathbf{q}, \mathbf{G}, \mathbf{G}^{\prime}$ as above for silicon. Our calculated diagonal element agrees with literature results [41-44]; in particular, a strongly bound exciton at $14.6 \mathrm{eV}$, well within the QP gap given by the onset of $\mathrm{RPA}+G W$, is observed in the measured spectra and correctly described by the BSE. The question is how the exciton will affect the off-diagonal elements. Our calculation shows that, as in the case of the diagonal, RPA $+G W$ yields a spectrum that is quite featureless. It is negative and of significant intensity, but more than a factor of 2 weaker than the diagonal one. Including now the electron-hole interaction, the structures in the off-diagonal element above $15 \mathrm{eV}$ reflect structures in the diagonal element with positive or negative sign and, as in the case of RPA $+G W$, clearly reduced intensity. Below the $G W$ gap also in the off-diagonal element a strongly bound exciton peak appears. Strikingly, it is approximately the specular negative of its diagonal counterpart at $14.6 \mathrm{eV}$, with a very similar intensity. This shows that it could be detrimental to neglect off-diagonal elements, for example, to get insight into bound excitons in real space [53].

The CIXS experiment is difficult, and published results are today still limited to the first pioneering works on silicon [60]. Our results, while showing the importance of an $a b$ initio theoretical approach with predictive power, strongly motivate a renewed experimental effort. At the same time, they can impact other fields of theory. Indeed, in principle the full $\chi$ including excitonic effects can also be calculated in TDDFT [76,77]. With respect to the two-particle BSE, TDDFT has the computational advantage of relying on the one-particle Kohn-Sham equation. It is therefore a widely used approach all over physics and chemistry [78-81]. In TDDFT, $\chi$ can be obtained from the linear response matrix equation $\chi=\chi^{\mathrm{RPA}}+\chi^{\mathrm{RPA}} f_{\mathrm{xc}} \chi$, where $\chi^{\mathrm{RPA}}$ is the RPA response function based on Kohn-Sham ingredients, and the exchange-correlation kernel $f_{\mathrm{xc}}$ is the functional derivative of the Kohn-Sham exchange-correlation potential with respect to the density [25]. As $\chi_{\mathbf{G G}^{\prime}}(\mathbf{q}, \omega)$, in a crystal $\chi^{\mathrm{RPA}}$ and $f_{\mathrm{xc}}$ are matrices in reciprocal space. Unfortunately, the widely used approximations for $f_{\mathrm{xc}}$, such as the ALDA, fail to describe excitonic effects in extended systems [82]. More advanced kernels have been derived from the BSE [43,8391] or modeled using related knowledge [92-98], but no known expression is to date able to well describe spectra

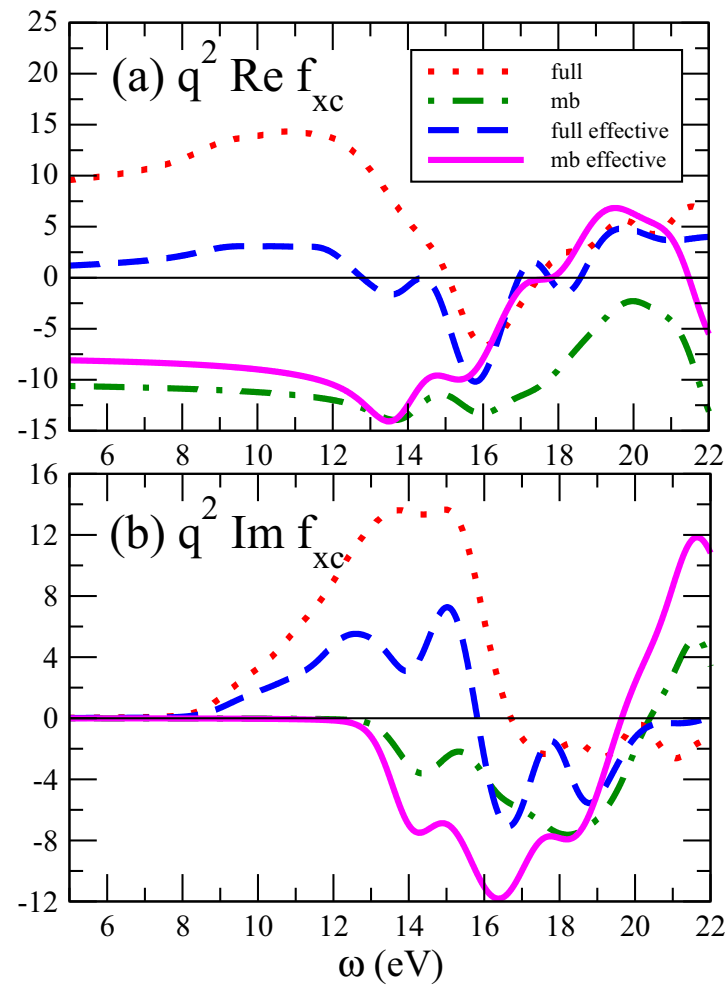

FIG. 2. Real (top panel) and imaginary (bottom panel) parts of exchange-correlation kernels of $\mathrm{LiF}$ for $\mathbf{q} \rightarrow \mathbf{0}: \mathbf{G}=\mathbf{G}^{\prime}=\mathbf{0}$ element of $f_{\mathrm{xc}}$ (red dotted line) and of $f_{\mathrm{xc}}^{\mathrm{mb}}$ (green dotted-dashed line), together with the effective scalar kernels $f_{\mathrm{xc}}^{\text {eff }}$ (blue dashed line) and $f_{\mathrm{xc}}^{\mathrm{mb}}$ eff (magenta solid line) that simulate absorption spectra (see text for definitions).

including bound and continuum excitons with a reasonable computational effort.

One obstacle for approximating $f_{\mathrm{xc}}$ is the fact that little is known, in particular, concerning off-diagonal elements. If $\chi$ was given, in principle $f_{\mathrm{xc}}$ could be studied by inverting the linear response equation, $f_{\mathrm{xcG}_{\mathbf{G}}}(\mathbf{q}, \omega)=\left(\chi^{\mathrm{RPA}}\right)_{\mathbf{G G}^{\prime}}^{-1}(\mathbf{q}, \omega)-$ $\chi_{\mathbf{G G}^{\prime}}^{-1}(\mathbf{q}, \omega)$. However, up to now off-diagonal elements of $\chi_{\mathbf{G G}^{\prime}}(\mathbf{q}, \omega)$ in the presence of excitonic effects were unknown, and as Fig. 1 shows, they cannot be neglected.

The present work overcomes this difficulty, since we now have the full matrix $\chi_{\mathbf{G G}^{\prime}}(\mathbf{q}, \omega)$ and can therefore invert the linear response equation. In line with literature, we will distinguish two definitions: the $f_{\mathrm{xc}}$ from TDDFT as explained above, and $f_{\mathrm{xcG}}^{\mathrm{mb}}\left(\mathbf{G} \mathbf{G}^{\prime}, \omega\right) \equiv\left(\chi^{\mathrm{RPA}+G W}\right)_{\mathbf{G G}^{\prime}}^{-1}(\mathbf{q}, \omega)-$ $\chi_{\mathbf{G G}^{\prime}}^{-1}(\mathbf{q}, \omega)$. The latter "many-body" kernel does not have the difficult task to open the quasiparticle gap, because $\chi^{\mathrm{RPA}+G W}$ is built with the $G W$ band structure. It is therefore a common starting point for successful model kernels (all kernels in Refs. [43,83-89,92-96] use this approach). In particular, we can address the following questions: What is the global structure of these kernels? Are there significant differences that might make one or the other easier to model? Can one detect a special feature linked to the bound exciton?

Figure 2(a) shows for LiF the real part of the $\mathbf{q} \rightarrow \mathbf{0}$ head elements $\mathbf{G}=\mathbf{G}^{\prime}=\mathbf{0}$ of $f_{\mathrm{xc}}$ and $f_{\mathrm{xc}}^{\mathrm{mb}}$. Both kernels have a pronounced frequency dependence. For this reason, the real 


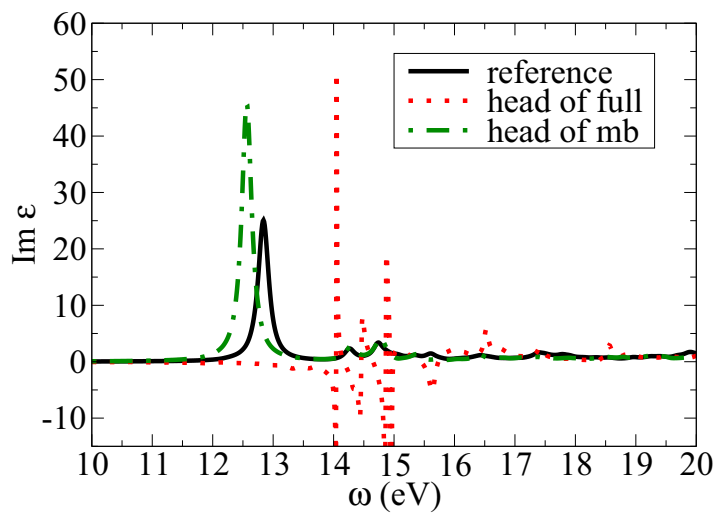

FIG. 3. LiF, absorption spectrum. The reference spectrum in black is the result of a BSE calculation, which is by definition, and numerically, identical to results obtained in TDDFT using $f_{\mathrm{xc}}^{\text {eff }}$. Results obtained by using only the head of the matrix $f_{\mathrm{xc}}$, and of the matrix $f_{\mathrm{xc}}^{\mathrm{mb}}$ are shown in red dotted and green dotted-dashed lines, respectively.

parts must be accompanied by a respective imaginary part, shown in Fig. 2(b). For both $f_{\mathrm{xc}}$ and $f_{\mathrm{xc}}^{\mathrm{mb}}$ the imaginary parts are zero at low frequency. The onset of intensity is above $8 \mathrm{eV}$ for $f_{\mathrm{xc}}$, but only above $12 \mathrm{eV}$ for $f_{\mathrm{xc}}^{\mathrm{mb}}$. The reason lies in the fact that $\operatorname{Im} f_{\mathrm{xc}}$ has to destroy intensity in the absorption spectrum between the onset of the Kohn-Sham spectrum and the bound exciton, whereas $f_{\mathrm{xc}}^{\mathrm{mb}}$ only has to create the bound exciton in the QP gap that is already open in $\chi^{\mathrm{RPA}+G W}$ [99]. The two kernels $f_{\mathrm{xc}}$ and $f_{\mathrm{xc}}^{\mathrm{mb}}$ start with opposite sign, both in the real and in the imaginary part, reflecting the fact that their task is to transfer oscillator strength to higher or lower energy, respectively.

Our calculations allow us to estimate the importance of the matrix components of the kernels beyond the only head. To this aim, Fig. 3 shows absorption spectra evaluated from $\operatorname{Im} \epsilon(\omega)=\operatorname{Im}\left[1+v_{c} \chi(\mathbf{q} \rightarrow \mathbf{0}, \omega)\right]_{\mathbf{G}=\mathbf{G}^{\prime}=\mathbf{0}}^{-1}$, where $\chi_{\mathbf{G G}^{\prime}}(\mathbf{q}, \omega)$ is calculated taking into account either the full matrix $f_{\mathrm{xc}}$ or $f_{\mathrm{xc}}^{\mathrm{mb}}$ (which both, by definition and also in practice, yield the reference BSE result and are therefore not shown), or only the respective head. The reference spectrum is the black line. Using only the head of the matrix $f_{\mathrm{xc}}$ leads to a disaster, with a spectrum that is washed out by oscillations, with even negative spectral weight, and the complete absence of a bound exciton. Restricting the matrix to the only head element in $f_{\mathrm{xc}}^{\mathrm{mb}}$, instead, has a less dramatic effect: In particular, the spectral weight remains mainly positive, and the strongly bound exciton appears and is reasonably well positioned. This is a clear indication for the fact that it is much easier to model $f_{\mathrm{xc}}^{\mathrm{mb}}$ than $f_{\mathrm{xc}}$. Still, the excitonic transfer of spectral weight to lower energies is severely overestimated, suppressing the spectrum at higher energies, similar to results of model scalar and static kernels [93,100,101].

Alternatively, one can define effective scalar kernels $f_{\mathrm{xc}}^{\text {eff }}(\mathbf{q}, \omega) \equiv 1 / \chi_{00}^{\mathrm{RPA}}(\mathbf{q}, \omega)-1 / \chi_{00}(\mathbf{q}, \omega) \quad$ and $f_{\mathrm{xc}}^{\mathrm{mb} \text { eff }}(\mathbf{q}, \omega) \equiv 1 / \chi_{00}^{\mathrm{RPA}+G W}(\mathbf{q}, \omega)-1 / \chi_{00}(\mathbf{q}, \omega)$ [102] that yield by definition the absorption spectrum. For silicon, the shape of our $f_{\mathrm{xc}}^{\mathrm{mb} \text { eff }}$ compares well with Ref. [102]. Here, instead, we explore a strongly bound exciton, with the example of LiF shown in Fig. 2. The effective kernels show similar structures as the head elements of their matrix version. However, the amplitude of both real and imaginary part [103] is smaller for the effective $f_{\mathrm{xc}}^{\text {eff }}$ than for the head of $f_{\mathrm{xc}}$. This means that off-diagonal elements in the full response equation compensate to some extent. Altogether, our results suggest to direct efforts towards the modeling of $\mathrm{mb}$ or of effective scalar, rather than full, kernels for spectroscopy.

In conclusion, we have shown that the off-diagonal elements of the mixed dynamic structure factor carry important excitonic effects below and above the quasiparticle gap. This insight could be gained owing to a generalization of the stateof-the-art $a b$ initio solution of the Bethe-Salpeter equation. Our calculation of the continuum excitonic effects in silicon bring the spectra of off-diagonal elements in good agreement with experiments. Moreover, we predict that the strongly bound exciton in LiF that is seen in optics, EELS and IXS should also be observable in some off-diagonal elements of the MDSF. These off-diagonal elements have an intensity that is comparable to the intensity of diagonal elements to which experiments are usually limited, and cannot be neglected when one wants to access the full spatially resolved density response. This motivates a renewed experimental effort, especially concerning coherent IXS. Having access to the full response matrices also gives new theoretical impulses. In particular, it allowed us to derive information concerning various flavors of effective exchange-correlation kernels in the framework of time-dependent density functional theory, suggesting the ones most amenable to simple approximations.

This research was supported by the European Research Council (ERC Grant Agreement No. 320971) and by a Marie Curie FP7 Integration Grant within the 7th European Union Framework Programme. This work was performed using HPC resources from GENCI-TGCC (Grant No. 2018-544).
[1] P. Nozieres and D. Pines, Theory of Quantum Liquids, Advanced Books Classics (Avalon, New York, 1999).

[2] G. Giuliani and G. Vignale, Quantum Theory of the Electron Liquid (Cambridge University Press, Cambridge, UK, 2005).

[3] S. Botti and M. Gatti, in Fundamentals of Time-Dependent Density Functional Theory, edited by M. A. Marques, N. T. Maitra, F. M. Nogueira, E. Gross, and A. Rubio (Springer, Berlin, 2012), pp. 29-50.

[4] R. Egerton, Electron Energy-Loss Spectroscopy in the Electron Microscope (Plenum, New York, 1986).
[5] G. G. Macfarlane, T. P. McLean, J. E. Quarrington, and V. Roberts, Phys. Rev. 111, 1245 (1958).

[6] Handbook of Ellipsometry, edited by H. G. Tompkins and E. A. Irene (William Andrew, Norwich, NY, 2005).

[7] W. Schülke, Electron Dynamics by Inelastic X-Ray Scattering, Oxford Series on Synchrotron Radiation (Oxford University Press, Oxford, UK, 2007).

[8] K. S. Song and R. T. Williams, Self-Trapped Excitons, Springer Series in Solid-State Sciences (Springer, Berlin, 1993). 
[9] R. M. Martin, L. Reining, and D. M. Ceperley, Interacting Electrons (Cambridge University Press, Cambridge, UK, 2016).

[10] L. Hedin, Phys. Rev. 139, A796 (1965).

[11] J. Lindhard, K. Dan. Vidensk. Selsk. Mat. Fys. Medd. 28, 1 (1954).

[12] D. Bohm and D. Pines, Phys. Rev. 92, 609 (1953).

[13] S. Waidmann, M. Knupfer, B. Arnold, J. Fink, A. Fleszar, and W. Hanke, Phys. Rev. B 61, 10149 (2000).

[14] A. G. Marinopoulos, L. Reining, V. Olevano, A. Rubio, T. Pichler, X. Liu, M. Knupfer, and J. Fink, Phys. Rev. Lett. 89, 076402 (2002).

[15] V. M. Silkin, A. Balassis, P. M. Echenique, and E. V. Chulkov, Phys. Rev. B 80, 054521 (2009).

[16] Y. Q. Cai, P. C. Chow, O. D. Restrepo, Y. Takano, K. Togano, H. Kito, H. Ishii, C. C. Chen, K. S. Liang, C. T. Chen, S. Tsuda, S. Shin, C. C. Kao, W. Ku, and A. G. Eguiluz, Phys. Rev. Lett. 97, 176402 (2006).

[17] R. Hambach, C. Giorgetti, N. Hiraoka, Y. Q. Cai, F. Sottile, A. G. Marinopoulos, F. Bechstedt, and L. Reining, Phys. Rev. Lett. 101, 266406 (2008).

[18] B. C. Larson, W. Ku, J. Z. Tischler, C.-C. Lee, O. D. Restrepo, A. G. Eguiluz, P. Zschack, and K. D. Finkelstein, Phys. Rev. Lett. 99, 026401 (2007).

[19] N. Hiraoka, M. Suzuki, K. D. Tsuei, H. Ishii, Y. Q. Cai, M. W. Haverkort, C. C. Lee, and W. Ku, Europhys. Lett. 96, 37007 (2011).

[20] C.-C. Lee, H. C. Hsueh, and W. Ku, Phys. Rev. B 82, 081106(R) (2010)

[21] F. Iori, F. Rodolakis, M. Gatti, L. Reining, M. Upton, Y. Shvyd'ko, J.-P. Rueff, and M. Marsi, Phys. Rev. B 86, 205132 (2012).

[22] H.-C. Weissker, J. Serrano, S. Huotari, F. Bruneval, F. Sottile, G. Monaco, M. Krisch, V. Olevano, and L. Reining, Phys. Rev. Lett. 97, 237602 (2006).

[23] M. Cazzaniga, H.-C. Weissker, S. Huotari, T. Pylkkänen, P. Salvestrini, G. Monaco, G. Onida, and L. Reining, Phys. Rev. B 84, 075109 (2011).

[24] S. Huotari, M. Cazzaniga, H.-C. Weissker, T. Pylkkänen, H. Müller, L. Reining, G. Onida, and G. Monaco, Phys. Rev. B 84, 075108 (2011).

[25] G. Onida, L. Reining, and A. Rubio, Rev. Mod. Phys. 74, 601 (2002).

[26] R. S. Knox, Theory of Excitons (Academic, New York, 1963).

[27] F. Bassani and G. P. Parravicini, Electronic States and Optical Transitions in Solids (Pergamon, Oxford, UK, 1975).

[28] E. E. Salpeter and H. A. Bethe, Phys. Rev. 84, 1232 (1951).

[29] G. Strinati, Riv. Nuovo Cimento 11, 1 (1988).

[30] W. Hanke and L. J. Sham, Phys. Rev. Lett. 43, 387 (1979).

[31] W. Hanke and L. J. Sham, Phys. Rev. B 21, 4656 (1980).

[32] G. Strinati, Phys. Rev. Lett. 49, 1519 (1982).

[33] G. Strinati, Phys. Rev. B 29, 5718 (1984).

[34] G. Onida, L. Reining, R. W. Godby, R. Del Sole, and W. Andreoni, Phys. Rev. Lett. 75, 818 (1995).

[35] S. Albrecht, L. Reining, R. Del Sole, and G. Onida, Phys. Rev. Lett. 80, 4510 (1998).

[36] L. X. Benedict, E. L. Shirley, and R. B. Bohn, Phys. Rev. Lett. 80, 4514 (1998).

[37] M. Rohlfing and S. G. Louie, Phys. Rev. Lett. 81, 2312 (1998).

[38] V. Olevano and L. Reining, Phys. Rev. Lett. 86, 5962 (2001).
[39] B. Arnaud, S. Lebègue, and M. Alouani, Phys. Rev. B 71, 035308 (2005).

[40] M. Palummo, O. Pulci, A. Marini, L. Reining, and R. Del Sole, Phys. Rev. B 74, 235431 (2006).

[41] W. A. Caliebe, J. A. Soininen, E. L. Shirley, C.-C. Kao, and K. Hämäläinen, Phys. Rev. Lett. 84, 3907 (2000).

[42] J. A. Soininen and E. L. Shirley, Phys. Rev. B 61, 16423 (2000).

[43] A. Marini, R. Del Sole, and A. Rubio, Phys. Rev. Lett. 91, 256402 (2003).

[44] M. Gatti and F. Sottile, Phys. Rev. B 88, 155113 (2013).

[45] T. Sander, E. Maggio, and G. Kresse, Phys. Rev. B 92, 045209 (2015).

[46] G. Fugallo, M. Aramini, J. Koskelo, K. Watanabe, T. Taniguchi, M. Hakala, S. Huotari, M. Gatti, and F. Sottile, Phys. Rev. B 92, 165122 (2015).

[47] P. Cudazzo, L. Sponza, C. Giorgetti, L. Reining, F. Sottile, and M. Gatti, Phys. Rev. Lett. 116, 066803 (2016).

[48] D. Y. Qiu, T. Cao, and S. G. Louie, Phys. Rev. Lett. 115, 176801 (2015).

[49] J. Koskelo, G. Fugallo, M. Hakala, M. Gatti, F. Sottile, and P. Cudazzo, Phys. Rev. B 95, 035125 (2017).

[50] J. Vinson, J. J. Rehr, J. J. Kas, and E. L. Shirley, Phys. Rev. B 83, 115106 (2011).

[51] P. Abbamonte, K. D. Finkelstein, M. D. Collins, and S. M. Gruner, Phys. Rev. Lett. 92, 237401 (2004).

[52] P. Abbamonte, G. C. L. Wong, D. G. Cahill, J. P. Reed, R. H. Coridan, N. W. Schmidt, G. H. Lai, Y. I. Joe, and D. Casa, Adv. Mater. 22, 1141 (2010).

[53] P. Abbamonte, T. Graber, J. P. Reed, S. Smadici, C.-L. Yeh, A. Shukla, J.-P. Rueff, and W. Ku, Proc. Natl. Acad. Sci. USA 105, 12159 (2008).

[54] Y. Gan, A. Kogar, and P. Abbamonte, Chem. Phys. 414, 160 (2013).

[55] T. Iwashita, B. Wu, W.-R. Chen, S. Tsutsui, A. Q. R. Baron, and T. Egami, Sci. Adv. 3, e1603079 (2017).

[56] S. L. Adler, Phys. Rev. 126, 413 (1962).

[57] N. Wiser, Phys. Rev. 129, 62 (1963).

[58] M. Ehrnsperger and H. Bross, J. Phys.: Condens. Matter 9. 1225 (1997).

[59] V. Olevano, Proprietà dielettriche di semiconduttori oltre l'approssimazione di densità locale, Ph.D. thesis, Università degli Studi di Roma “Tor Vergata," 1999.

[60] W. Schülke and A. Kaprolat, Phys. Rev. Lett. 67, 879 (1991).

[61] H.-C. Weissker, J. Serrano, S. Huotari, E. Luppi, M. Cazzaniga, F. Bruneval, F. Sottile, G. Monaco, V. Olevano, and L. Reining, Phys. Rev. B 81, 085104 (2010).

[62] J. R. Fields, P. C. Gibbons, and S. E. Schnatterly, Phys. Rev. Lett. 38, 430 (1977).

[63] H. Kohl and H. Rose, Adv. Electron. Electron Phys. 65, 173 (1985).

[64] P. Schattschneider, B. Jouffrey, and M. Nelhiebel, Phys. Rev. B 54, 3861 (1996).

[65] P. Schattschneider, S. Rubino, C. Hebert, J. Rusz, J. Kunes, P. Novák, E. Carlino, M. Fabrizioli, G. Panaccione, and G. Rossi, Nature (London) 441, 486 (2006)

[66] W. Schülke, Phys. Lett. A 83, 451 (1981).

[67] W. Schülke, Solid State Commun. 43, 863 (1982).

[68] W. Schülke and S. Mourikis, Acta Crystallogr., Sect. A 42, 86 (1986). 
[69] W. M. Saslow and G. F. Reiter, Phys. Rev. B 7, 2995 (1973).

[70] R. Girlanda, M. Parrinello, and E. Tosatti, Phys. Rev. Lett. 36, 1386 (1976).

[71] L. E. Oliveira and K. Sturm, Phys. Rev. B 22, 6283 (1980).

[72] K. Sturm and W. Schülke, Phys. Rev. B 46, 7193 (1992).

[73] See Supplemental Material at http://link.aps.org/supplemental/ 10.1103/PhysRevResearch.1.032010 for general formulation of theory and computational details.

[74] The experimental spectrum from Ref. [60] has been shifted by $2 \mathrm{eV}$ as in Ref. [61].

[75] M. Rohlfing and S. G. Louie, Phys. Rev. B 62, 4927 (2000).

[76] E. Runge and E. K. U. Gross, Phys. Rev. Lett. 52, 997 (1984).

[77] C. Ullrich, Time-Dependent Density-Functional Theory: Concepts and Applications, Oxford Graduate Texts (Oxford University Press, Oxford, UK, 2012).

[78] F. Furche and K. Burke, in Annual Reports in Computational Chemistry, edited by D. Spellmeyer, 1 st ed. (Elsevier, Amsterdam, 2005), Vol. 1, pp. 19-30.

[79] M. Casida and M. Huix-Rotllant, Annu. Rev. Phys. Chem. 63, 287 (2012).

[80] A. D. Laurent and D. Jacquemin, Int. J. Quantum Chem. 113, 2019 (2013).

[81] N. T. Maitra, J. Chem. Phys. 144, 220901 (2016).

[82] S. Botti, A. Schindlmayr, R. D. Sole, and L. Reining, Rep. Prog. Phys. 70, 357 (2007).

[83] L. Reining, V. Olevano, A. Rubio, and G. Onida, Phys. Rev. Lett. 88, 066404 (2002).

[84] S. Botti, F. Sottile, N. Vast, V. Olevano, L. Reining, H.-C. Weissker, A. Rubio, G. Onida, R. Del Sole, and R. W. Godby, Phys. Rev. B 69, 155112 (2004).

[85] F. Sottile, V. Olevano, and L. Reining, Phys. Rev. Lett. 91, 056402 (2003).

[86] G. Adragna, R. Del Sole, and A. Marini, Phys. Rev. B 68, 165108 (2003).

[87] R. Stubner, I. V. Tokatly, and O. Pankratov, Phys. Rev. B 70, 245119 (2004).
[88] F. Bruneval, F. Sottile, V. Olevano, R. Del Sole, and L. Reining, Phys. Rev. Lett. 94, 186402 (2005).

[89] M. Gatti, V. Olevano, L. Reining, and I. V. Tokatly, Phys. Rev. Lett. 99, 057401 (2007).

[90] Z. H. Yang, F. Sottile, and C. A. Ullrich, Phys. Rev. B 92, 035202 (2015).

[91] U. von Barth, N. E. Dahlen, R. van Leeuwen, and G. Stefanucci, Phys. Rev. B 72, 235109 (2005).

[92] S. Sharma, J. K. Dewhurst, A. Sanna, and E. K. U. Gross, Phys. Rev. Lett. 107, 186401 (2011).

[93] S. Rigamonti, S. Botti, V. Veniard, C. Draxl, L. Reining, and F. Sottile, Phys. Rev. Lett. 114, 146402 (2015).

[94] Y.-M. Byun and C. A. Ullrich, Phys. Rev. B 95, 205136 (2017).

[95] P. E. Trevisanutto, A. Terentjevs, L. A. Constantin, V. Olevano, and F. D. Sala, Phys. Rev. B 87, 205143 (2013).

[96] J. A. Berger, Phys. Rev. Lett. 115, 137402 (2015).

[97] V. U. Nazarov, G. Vignale, and Y.-C. Chang, Phys. Rev. Lett. 102, 113001 (2009).

[98] V. U. Nazarov and G. Vignale, Phys. Rev. Lett. 107, 216402 (2011).

[99] The need for a nonzero imaginary part for the xc kernel to account for the band-gap opening has been demonstrated in Refs. [2,104].

[100] F. Sottile, K. Karlsson, L. Reining, and F. Aryasetiawan, Phys. Rev. B 68, 205112 (2003).

[101] Note that in the present work all kernels are frequency dependent.

[102] R. Del Sole, G. Adragna, V. Olevano, and L. Reining, Phys. Rev. B 67, 045207 (2003).

[103] Note that both the real and imaginary parts of the kernels are important to obtain the correct spectrum [73].

[104] E. K. U. Gross and N. T. Maitra, in Fundamentals of TimeDependent Density Functional Theory, edited by M. A. Marques, N. T. Maitra, F. M. Nogueira, E. Gross, and A. Rubio, Lecture Notes in Physics Vol. 837 (Springer, Berlin, 2012), p. 53. 